SUPPORTING INFORMATION

\title{
Proton Transfer Reaction Rates in Phenol-Ammonia Cluster Cation
}

Hiroto TACHIKAWA* and Tetsuji IYAMA

Division of Applied Chemistry, Faculty of Engineering,

Hokkaido University, Sapporo 060-8628, JAPAN

hiroto@eng.hokudai.ac.jp

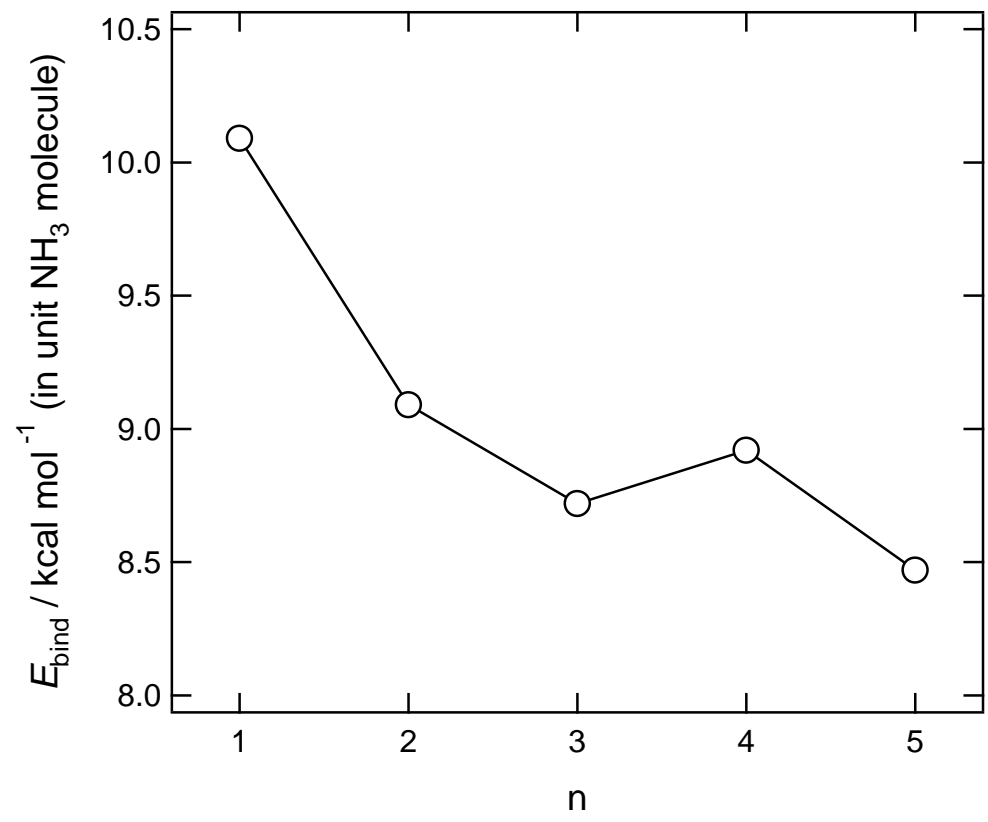

Figure S1. Binding energies of ammonia clusters to $\mathrm{PhOH}$ (per one $\mathrm{NH}_{3}$ molecule and in $\mathrm{kcal} / \mathrm{mol}): \mathrm{PhOH}+n\left(\mathrm{NH}_{3}\right) \rightarrow \mathrm{PhOH}-\left(\mathrm{NH}_{3}\right)_{\mathrm{n}}(n=1-5)$, calculated at the MP2/6-311++G(d,p) level. 

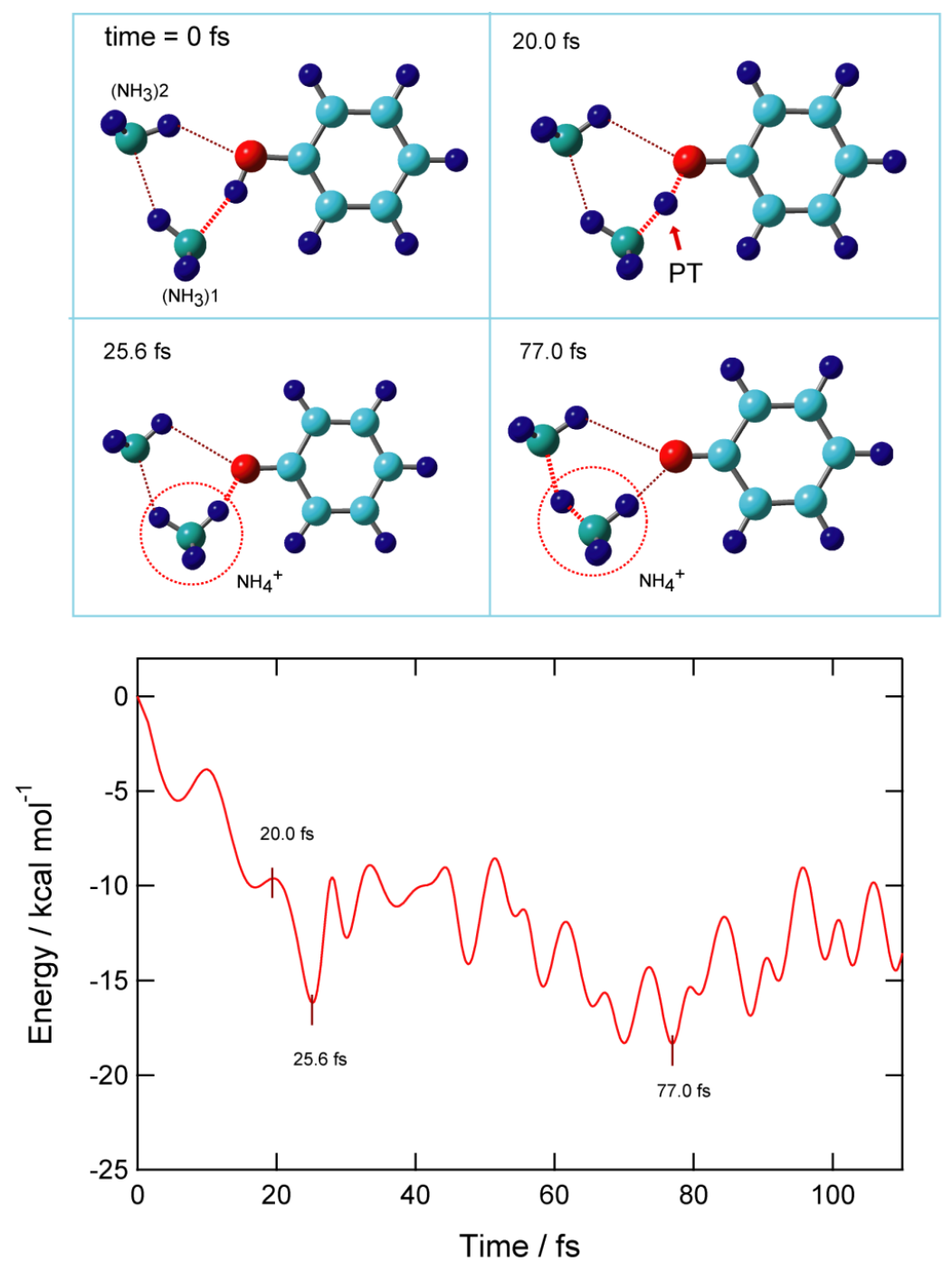

Figure S2. (Upper) Snapshots of $\mathrm{PhOH}^{+}-\left(\mathrm{NH}_{3}\right)_{\mathrm{n}}(n=2)$ after vertical ionization from neutral state calculated as a function of time. The direct AIMD calculation was carried out at the CAM-B3LYP/6-311G(d,p) level. (Lower) Time evolution of potential energy of $\mathrm{PhOH}^{+}-\left(\mathrm{NH}_{3}\right)_{\mathrm{n}}(n=2)$. 

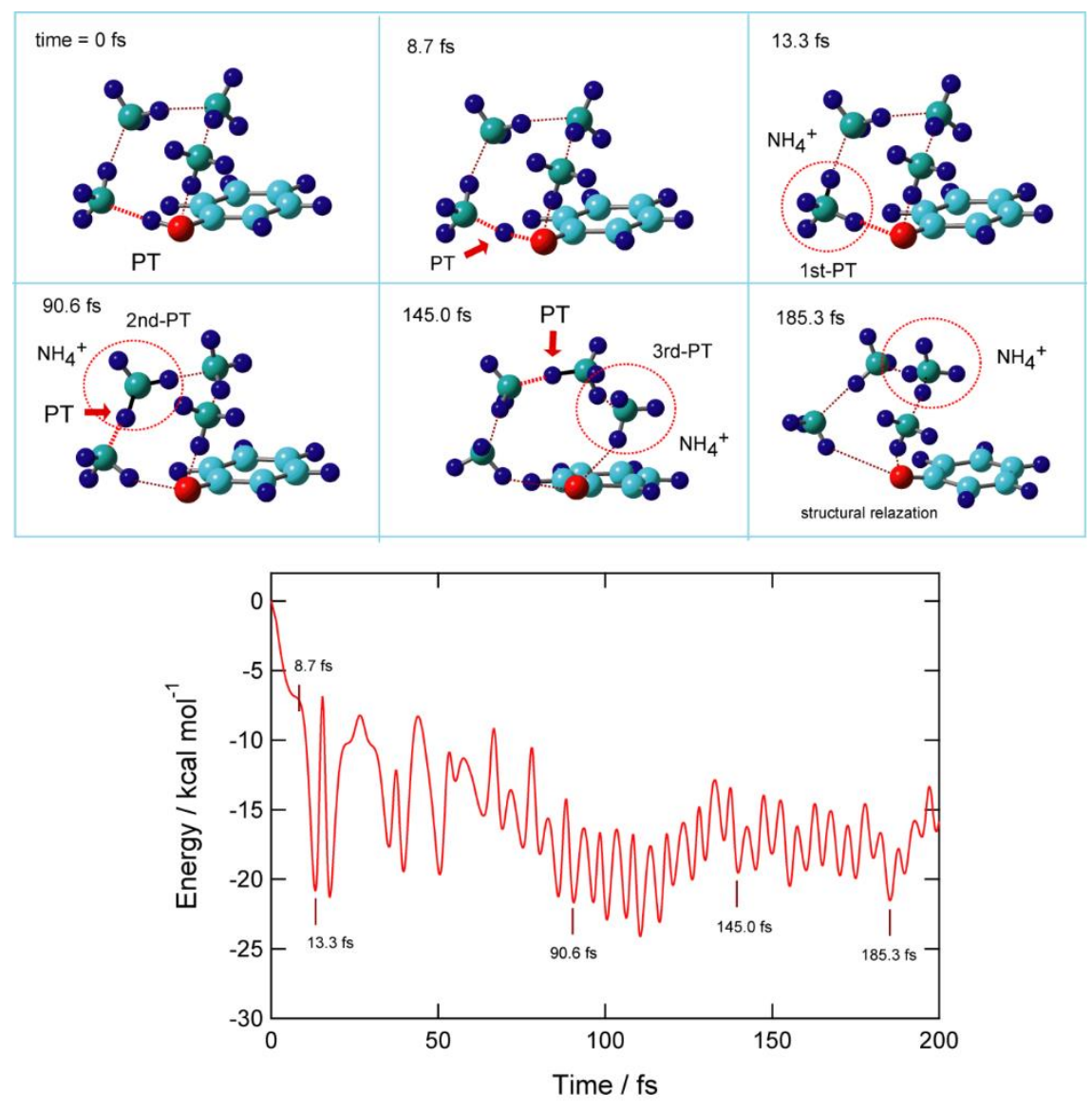

Figure S3. (Upper) Snapshots of $\mathrm{PhOH}^{+}-\left(\mathrm{NH}_{3}\right)_{\mathrm{n}}(n=4)$ after vertical ionization from neutral state calculated as a function of time. The direct AIMD calculation was carried out at the CAM-B3LYP/6-311G(d,p) level. (Lower) Time evolution of potential energy of $\mathrm{PhOH}^{+}-\left(\mathrm{NH}_{3}\right)_{\mathrm{n}}(n=4)$. 


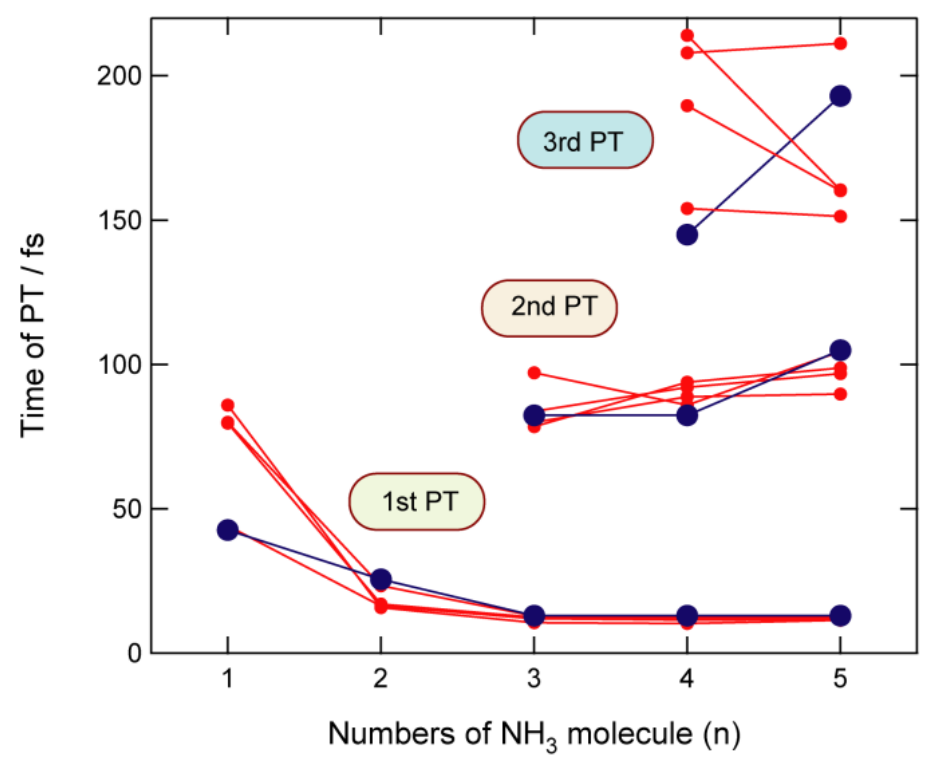

Figure S4. Time of proton transfer $(\mathrm{PT})$ for $\mathrm{PhOH}-\left(\mathrm{NH}_{3}\right)_{\mathrm{n}}(n=1-5)$ plotted as a function of cluster size $(n)$. First, second, and third PTs are given. The calculations were carried out using sets A, B, C, D, and S.

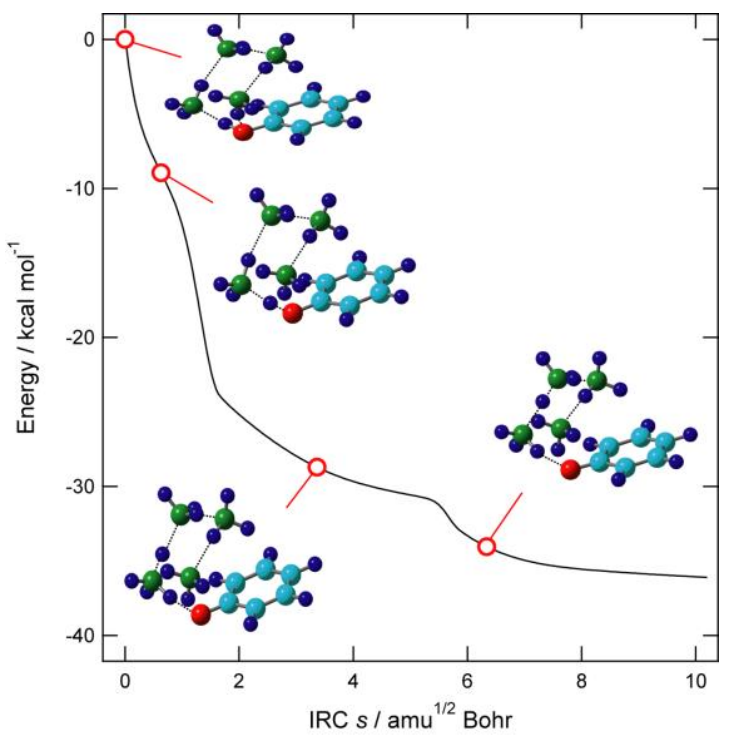

Figure S5. Intrinsic reaction coordinate (IRC) of $\mathrm{PhOH}^{+}-\left(\mathrm{NH}_{3}\right)_{\mathrm{n}}(n=4)$ after the ionization of neutral cluster. The calculation was carried out at the CAM-B3LYP/6-31G(d) level. 


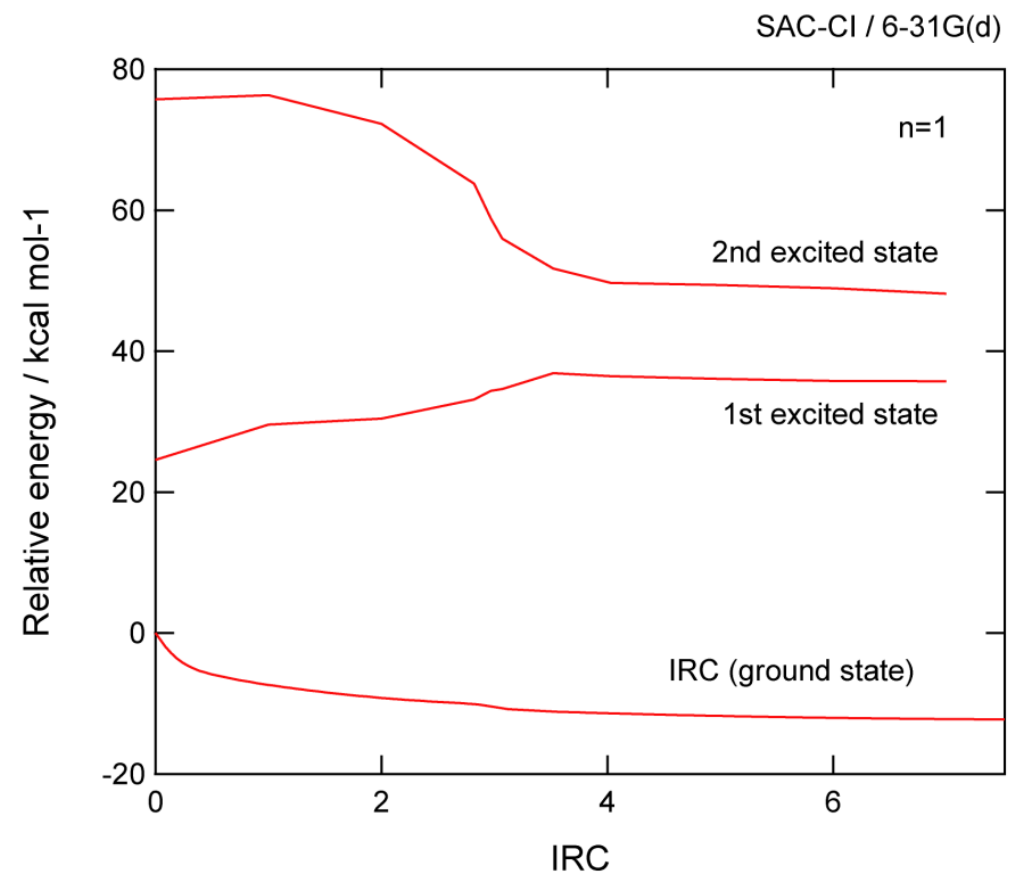

Figure 6S. Potential energy curves for ground, first and second excited states plotted as a function of IRC in $\mathrm{PhOH}^{+}-\mathrm{NH}_{3}$. The calculations were carried out at the SAC-CI/ 6-31G(d) level.

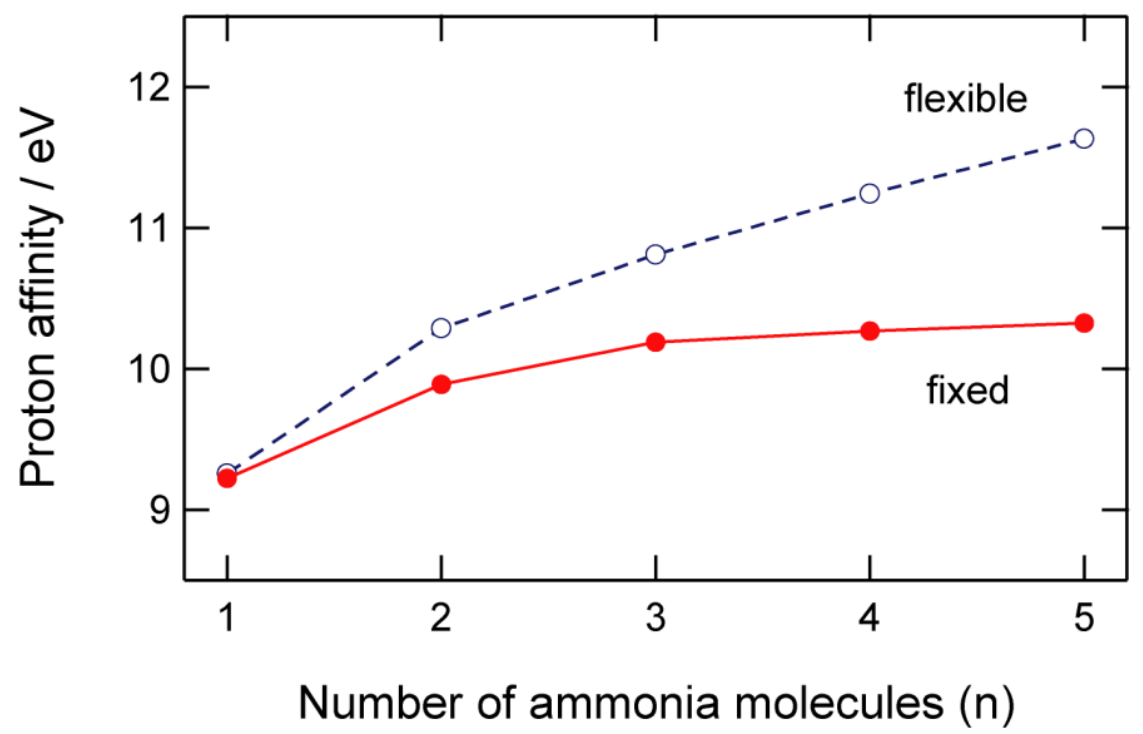

Figure S7. Proton affinities of ammonia clusters $\left(\mathrm{NH}_{3}\right)_{\mathrm{n}}(n=1-5)$ plotted as a function of cluster size $(n)$ (See text). 


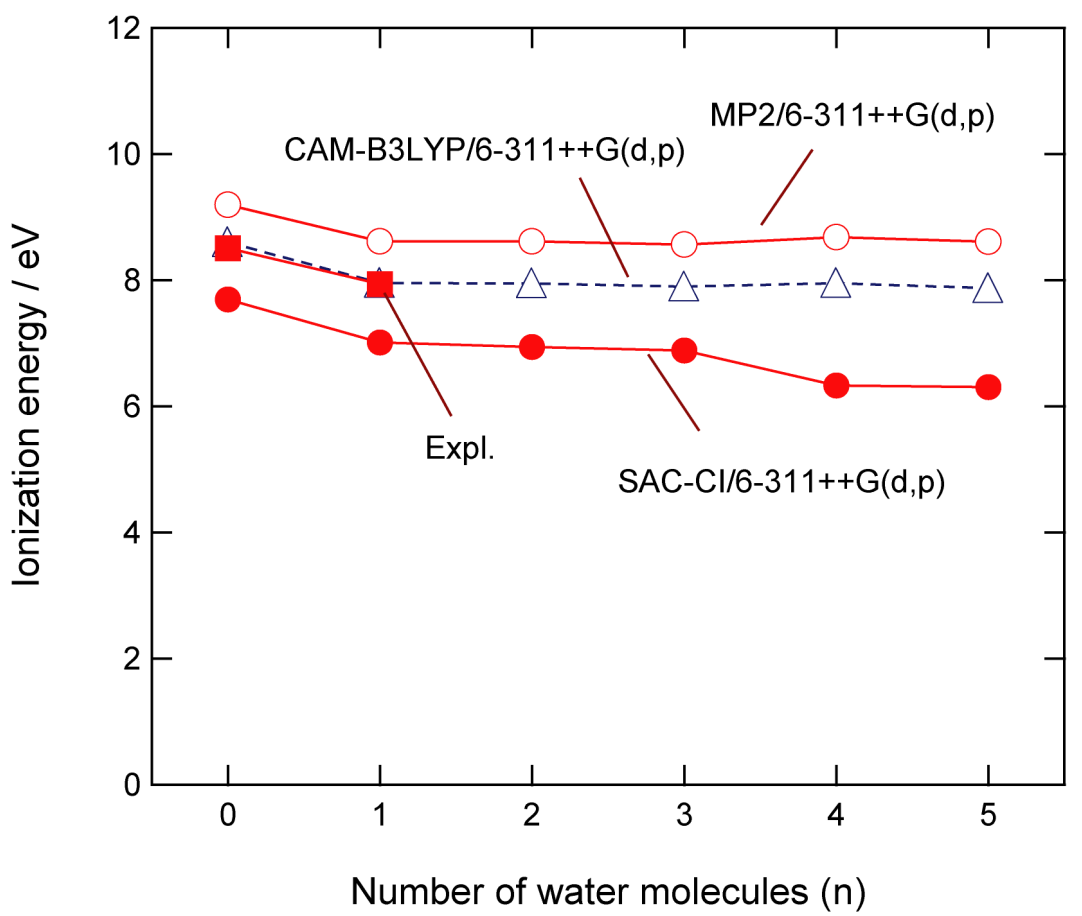

Figure S8. Vertical ionization energies of phenol monomer $(n=0)$ phenol-ammonia clusters $\mathrm{PhOH}-\left(\mathrm{NH}_{3}\right)_{\mathrm{n}}(n=1-5)$ plotted as a function of cluster size $(n)$. The calculations were carried out at MP2, CAM-B3LYP, and MP2 methods with $6-311++\mathrm{G}(\mathrm{d}, \mathrm{p})$ basis. Experimental values are cited from reference 31 . 
Table S1. Bond lengths and intermolecular distances in PhOH- $\left(\mathrm{NH}_{3}\right)_{\mathrm{n}}(n=1-5)$ (in $\AA$ ). The structures were optimized at the MP2/6-311++G(d,p)(denoted by MP2++G), CAM-B3LYP/ 6-311++G(d,p) (CAM++G), and CAM-B3LYP/6-311G(d,p) (CAMG) levels.

\begin{tabular}{|c|c|c|c|c|c|}
\hline \multicolumn{6}{|c|}{$\mathrm{MP} 2++\mathrm{G}$} \\
\hline & $n=1$ & $n=2$ & $n=3$ & $n=4$ & $n=5$ \\
\hline$r 1$ & 0.98102 & 0.98931 & 0.99705 & 0.99960 & 1.00039 \\
\hline R1 & 1.86641 & 1.79495 & 1.74502 & 1.73349 & 1.72140 \\
\hline$r 2$ & & 1.02322 & 1.02812 & 1.02924 & 1.02790 \\
\hline $\mathrm{R} 2$ & & 2.19569 & 2.07240 & 2.05248 & 2.07211 \\
\hline r3 & & 1.01816 & 1.02425 & 1.02771 & 1.02735 \\
\hline R3 & & 2.24307 & 2.14785 & 2.09419 & 2.11115 \\
\hline$r 4$ & & & 1.01961 & 1.02257 & 1.02712 \\
\hline R4 & & & 2.08143 & 2.20108 & 2.11243 \\
\hline r5 & & & & 1.01941 & 1.02254 \\
\hline R5 & & & & 2.12825 & 2.19216 \\
\hline $\mathrm{Rh}$ & & & & 2.87218 & 2.81469 \\
\hline r6 & & & & & 1.01991 \\
\hline $\mathrm{R} 6$ & & & & & 2.07469 \\
\hline \multicolumn{6}{|c|}{$\mathrm{CAM}++\mathrm{G}$} \\
\hline & $n=1$ & $n=2$ & $n=3$ & $n=4$ & $n=5$ \\
\hline$r 1$ & 0.98212 & 0.99156 & 0.99977 & 1.00179 & 1.00211 \\
\hline R1 & 1.85901 & 1.79006 & 1.74201 & 1.73320 & 1.73042 \\
\hline r2 & & 1.02436 & 1.02993 & 1.03048 & 1.03078 \\
\hline $\mathrm{R} 2$ & & 2.15212 & 2.04364 & 2.03381 & 2.03483 \\
\hline r3 & & 1.01793 & 1.02582 & 1.02825 & 1.02820 \\
\hline R3 & & 2.20318 & 2.10998 & 2.07315 & 2.08169 \\
\hline$r 4$ & & & 1.01995 & 1.02360 & 1.02777 \\
\hline $\mathrm{R} 4$ & & & 2.05052 & 2.15429 & 2.08548 \\
\hline r5 & & & & 1.01944 & 1.02315 \\
\hline R5 & & & & 2.10663 & 2.16468 \\
\hline $\mathrm{Rh}$ & & & & 3.93135 & 3.34983 \\
\hline r6 & & & & & 1.01941 \\
\hline R6 & & & & & 2.10127 \\
\hline \multicolumn{6}{|c|}{ CAMG } \\
\hline & $n=1$ & $n=2$ & $n=3$ & $n=4$ & $n=5$ \\
\hline r1 & 0.98288 & 0.99383 & 1.00310 & 1.00543 & 1.00558 \\
\hline $\mathrm{R} 1$ & 1.83412 & 1.76921 & 1.71411 & 1.70440 & 1.70292 \\
\hline$r 2$ & & 1.02608 & 1.03326 & 1.03395 & 1.03487 \\
\hline $\mathrm{R} 2$ & & 2.08326 & 1.98132 & 1.96812 & 1.96203 \\
\hline r3 & & 1.01815 & 1.02779 & 1.03120 & 1.03180 \\
\hline R3 & & 2.20239 & 2.05225 & 2.00548 & 2.00347 \\
\hline$r 4$ & & & 1.01995 & 1.02584 & 1.03021 \\
\hline $\mathrm{R} 4$ & & & 2.02651 & 2.07791 & 2.02428 \\
\hline r5 & & & & 1.01944 & 1.02464 \\
\hline R5 & & & & 2.08601 & 2.08997 \\
\hline $\mathrm{Rh}$ & & & & 3.93839 & 3.00931 \\
\hline r6 & & & & & 1.01942 \\
\hline R6 & & & & & 2.07836 \\
\hline
\end{tabular}


Table S2. Mulliken molecular charges and spin densities on $\mathrm{PhOH}$ and ammonia molecules of $\left[\mathrm{PhOH}^{+}-\left(\mathrm{NH}_{3}\right)_{\mathrm{n}}\right]_{\mathrm{ver}}(n=1-5)$ at time zero. The values were calculated at the MP2/6-311++G(d,p) (denoted by MP2++G) and CAM-B3LYP/6-311G(d,p) (CAM_6311) levels.

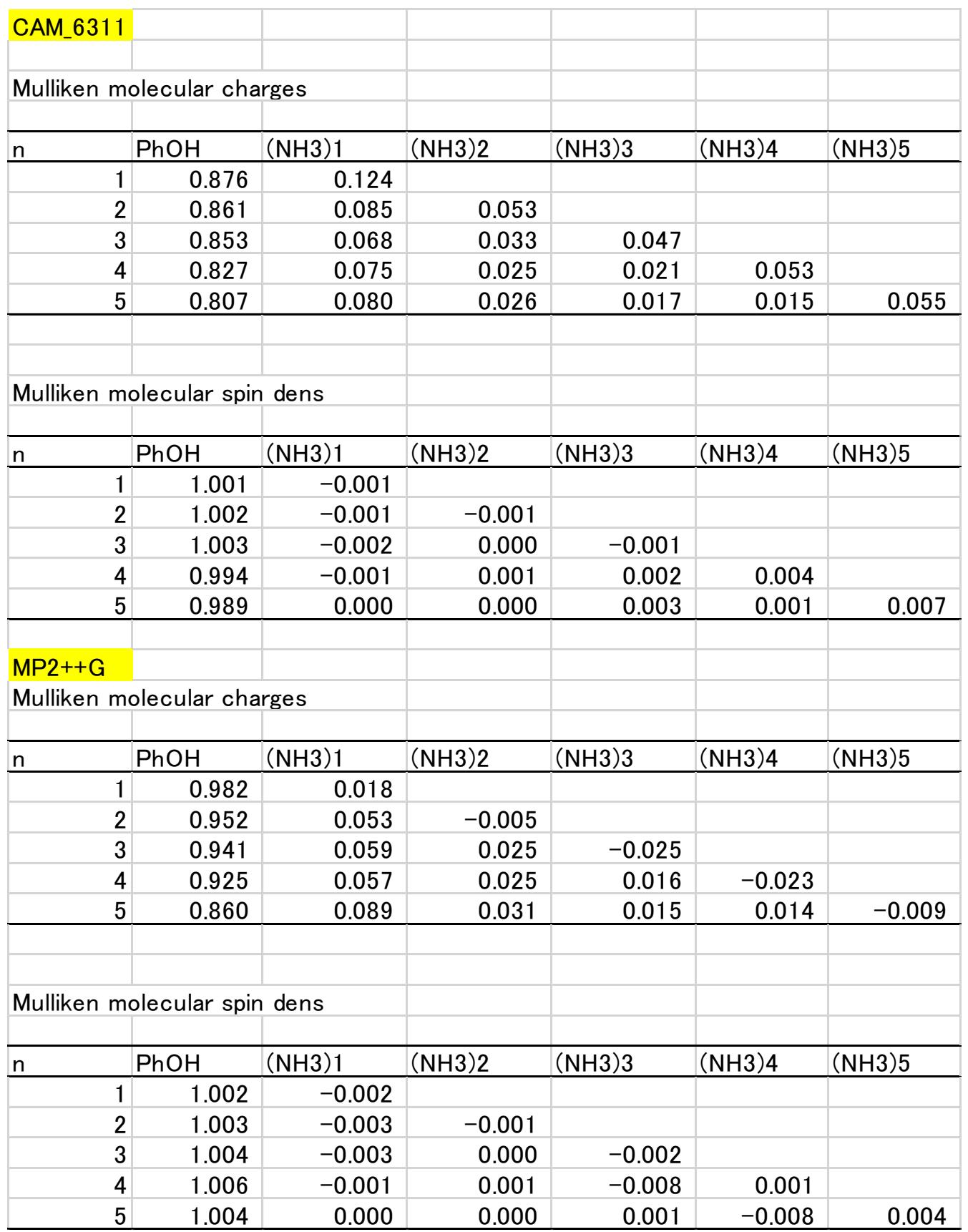


Table S3. Results of SAC-CI/6-311++G(d,p) calculations of $\left[\mathrm{PhOH}^{+}-\left(\mathrm{NH}_{3}\right)_{\mathrm{n}}\right]_{\mathrm{ver}}(n=$ 1-5) at time zero. Molecular charges on $\mathrm{PhOH}$ and ammonia molecules in $\left[\mathrm{PhOH}^{+}-\left(\mathrm{NH}_{3}\right)_{\mathrm{n}}\right]_{\text {ver }}(n=1-5)$ at ground and low-lying excited states are given. $\mathrm{E}$ (relative) means excitation energies from $\left[\mathrm{PhOH}^{+}-\left(\mathrm{NH}_{3}\right)_{\mathrm{n}}\right]_{\text {ver }}(n=1-5)($ in $\mathrm{eV})$.

\begin{tabular}{|c|c|c|c|c|c|}
\hline \multicolumn{6}{|l|}{$n=1$} \\
\hline & ground & 1st & 2nd & $3 r d$ & 4th \\
\hline $\mathrm{PhOH}$ & 0.992336 & 1.006100 & 0.709633 & 0.998470 & 0.521832 \\
\hline$(\mathrm{NH} 3) 1$ & 0.007663 & -0.006100 & 0.290366 & 0.001529 & 0.478168 \\
\hline \multicolumn{6}{|l|}{$n=2$} \\
\hline & ground & $1 \mathrm{st}$ & 2nd & $3 r d$ & 4th \\
\hline $\mathrm{PhOH}$ & 0.975211 & 0.995914 & 0.157313 & 0.064821 & 0.982144 \\
\hline$(\mathrm{NH} 3) 1$ & 0.031611 & 0.013649 & 0.594965 & 0.187388 & 0.025633 \\
\hline$(\mathrm{NH} 3) 2$ & -0.006819 & -0.009562 & 0.247723 & 0.747791 & -0.007777 \\
\hline \multicolumn{6}{|l|}{$n=3$} \\
\hline & ground & $1 \mathrm{st}$ & 2nd & $3 r d$ & 4th \\
\hline $\mathrm{PhOH}$ & 0.965203 & 0.987795 & 0.078478 & 0.067260 & 0.159733 \\
\hline$(\mathrm{NH} 3) 1$ & 0.023372 & 0.010736 & 0.184866 & 0.241455 & 0.271565 \\
\hline$(\mathrm{NH} 3) 2$ & 0.017962 & 0.017026 & 0.442651 & 0.061172 & 0.490375 \\
\hline$(\mathrm{NH} 3) 3$ & -0.006537 & -0.015556 & 0.294005 & 0.630113 & 0.078328 \\
\hline \multicolumn{6}{|l|}{$n=4$} \\
\hline & ground & 1st & 2nd & $3 r d$ & 4th \\
\hline $\mathrm{PhOH}$ & 0.958551 & 0.919575 & 0.088992 & 0.058216 & 0.070576 \\
\hline$(\mathrm{NH} 3) 1$ & 0.027836 & 0.009491 & -0.000205 & 0.145500 & 0.028192 \\
\hline$(\mathrm{NH} 3) 2$ & 0.020110 & 0.024456 & 0.192439 & 0.523465 & 0.033114 \\
\hline$(\mathrm{NH} 3) 3$ & 0.010350 & 0.064971 & 0.623344 & 0.171051 & 0.167324 \\
\hline$(\mathrm{NH} 3) 4$ & -0.016848 & -0.018494 & 0.095430 & 0.101767 & 0.700795 \\
\hline \multicolumn{6}{|l|}{$n=5$} \\
\hline & ground & $1 \mathrm{st}$ & 2nd & $3 r d$ & 4th \\
\hline $\mathrm{PhOH}$ & 0.896982 & 0.819725 & 0.054648 & -0.022252 & -0.006435 \\
\hline$(\mathrm{NH} 3) 1$ & 0.068927 & 0.050287 & 0.045215 & 0.052159 & 0.055359 \\
\hline$(\mathrm{NH} 3) 2$ & 0.018056 & 0.010656 & 0.043584 & 0.231476 & 0.073570 \\
\hline$(\mathrm{NH} 3) 3$ & 0.009788 & 0.023136 & 0.206805 & 0.394803 & 0.028191 \\
\hline$(\mathrm{NH} 3) 4$ & 0.015379 & 0.101954 & 0.553985 & 0.166623 & 0.202101 \\
\hline$(\mathrm{NH} 3) 5$ & -0.009133 & -0.005760 & 0.095765 & 0.177193 & 0.647213 \\
\hline
\end{tabular}


Figure S4. Time of proton transfer (in fs) for $\mathrm{PhOH}-\left(\mathrm{NH}_{3}\right)_{\mathrm{n}}(n=1-5)$ calculated at several levels of theory (sets S, and sets A-D). First, second, and third PT are given.

\begin{tabular}{|c|c|c|c|c|c|c|}
\hline & \multicolumn{3}{|c|}{ set-S } & \multicolumn{3}{c|}{ set-A } \\
\hline $\mathrm{n}$ & $1 \mathrm{st}$ & 2nd & 3rd & 1st & 2nd & 3rd \\
\hline 1 & 42.6 & & & 80.0 & & \\
\hline 2 & 25.6 & & & 23.3 & & \\
\hline 3 & 13.0 & 82.4 & & 12.9 & 83.8 & \\
\hline 4 & 13.0 & 82.4 & 145.0 & 12.7 & 92.1 & 154.0 \\
\hline 5 & 13.0 & 105.0 & 193.0 & 12.7 & 96.8 & 151.3 \\
\hline
\end{tabular}

\begin{tabular}{|c|c|c|c|c|c|c|}
\hline & \multicolumn{3}{|c|}{ set-B } & \multicolumn{3}{c|}{ set-C } \\
\hline $\mathrm{n}$ & $1 \mathrm{st}$ & 2nd & 3rd & 1st & 2nd & 3rd \\
\hline 1 & 79.7 & & & 44.1 & & \\
\hline 2 & 17.0 & & & 16.3 & & \\
\hline 3 & 12.4 & 97.1 & & 11.9 & 78.5 & \\
\hline 4 & 12.1 & 85.9 & 214.0 & 11.5 & 93.9 & 208.0 \\
\hline 5 & 12.3 & 104.5 & 160.5 & 11.7 & 98.8 & 211.2 \\
\hline
\end{tabular}

\begin{tabular}{|c|r|r|r|}
\hline & \multicolumn{3}{|c|}{ set-D } \\
\hline $\mathrm{n}$ & $1 \mathrm{st}$ & 2nd & 3rd \\
\hline 1 & 85.9 & & \\
\hline 2 & 15.8 & & \\
\hline 3 & 10.5 & 80.0 & \\
\hline 4 & 10.3 & 88.8 & 189.7 \\
\hline 5 & 11.4 & 89.8 & 160.2 \\
\hline
\end{tabular}


Table S5. Time of proton transfer (in fs) for PhOH- $\left(\mathrm{NH}_{3}\right)_{\mathrm{n}}(n=1-5)$ calculated from the initial geometries generated at $10 \mathrm{~K}$. Five trajectories were run from each size $(n)$.

\begin{tabular}{|c|r|r|r|r|}
\hline run & $\mathrm{n}=1$ & $\mathrm{n}=2$ & $\mathrm{n}=3$ (1st) & $\mathrm{n}=3$ (2nd) \\
\hline 1 & 45.2 & 16.1 & 10.3 & 80.6 \\
\hline 2 & 45.2 & 15.6 & 10.7 & 77.9 \\
\hline 3 & 44.7 & 16 & 11.2 & 78.9 \\
\hline 4 & 45.1 & 15.3 & 11.6 & 80.1 \\
\hline 5 & 43.9 & 16.2 & 11.7 & 79.9 \\
\hline 〈average〉 & 44.8 & 15.8 & 11.1 & 79.5 \\
\hline
\end{tabular}

\begin{tabular}{|c|r|r|r|r|r|r|}
\hline run & $\mathrm{n}=4$ (1st) & $\mathrm{n}=4$ (2nd) & $\mathrm{n}=4(3 \mathrm{rd})$ & $\mathrm{n}=5(1 \mathrm{st})$ & $\mathrm{n}=5(2 \mathrm{nd})$ & $\mathrm{n}=5(3 \mathrm{rd})$ \\
\hline 1 & 9.9 & 92 & 138.5 & 11.4 & 88.6 & 161.1 \\
\hline 2 & 9.9 & 87.8 & 191.1 & 11.2 & 95.4 & 155.7 \\
\hline 3 & 10.2 & 89.2 & 166 & 9.9 & 95.7 & 153.2 \\
\hline 4 & 11.5 & 92.9 & 180.3 & 10 & 92.3 & 171.2 \\
\hline 5 & 10.3 & 88.8 & 194.9 & 11.2 & 104.3 & 182 \\
\hline 〈average> & 10.4 & 90.1 & 174.2 & 10.7 & 95.3 & 164.6 \\
\hline
\end{tabular}

